\title{
PULMONARY CAPILLARY ARTERIAL PRESSURE PULSE IN MAN
}

\author{
BY \\ W. WEISSEL, F. SALZMANN, AND H. VETTER \\ From the II Medical University Clinic, Vienna \\ Received June 11, 1951
}

In 1948, Hellems, Haynes, Dexter, and Kinney, using venous and arterial catheterization in dogs obtained pressure recordings from both sides of the pulmonary capillary bed. The method consisted in wedging the tip of a cardiac catheter into a small branch of the pulmonary artery and of the pulmonary vein respectively, and recording the mean blood pressures in the obstructed vascular bed distal to the catheter tip. They felt that these pressures were "close approximations of the true capillary pressure" and the term capillary pressure is now widely used for the mean pressure obtainable by occluding a small branch of the pulmonary artery. Shortly afterwards, it was shown by Lagerloef and Werkoe (1949) that in man the tracings recorded on the arterial side of the capillary bed were of typical venous shape and closely resembled the tracings from the right (and left) atrium. They concluded that in the manometric system thus constructed not only capillary pulsations but also those from the venous side of the vascular bed were transmitted through the capillaries.

It seemed, therefore, of interest to investigate the type of curve obtainable by obstructing a small branch of the pulmonary vein. Accordingly this was done in five cases of atrial septal defect, and the tracings thus recorded resembled in many ways arterial pulsations. Since Lagerloef and Werkoe called their curves from the arterial side the " pulmonary capillary venous pressure pulse" (PCV) we should like to name the curves from the venous side of the capillary bed the " pulmonary capillary arterial pressure pulse" (PCA).

Methods. Cardiac catheterization was performed in the usual way. Cournand catheters No. F6-9 with the hole on the tip were used and connected with Tybjaerg-Hansen's capacitive manometer. Pressure tracings were recorded on Elmquist-triplex, together with electro- and phono-cardiograms. The damping of the manometer system was critical. In order to eliminate artefacts due to catheter movements, damping was further increased by the electrićal filter III on the amplifier. The mean pressures were highly overdamped curves using filter $\mathrm{V}$. The zero point for all pressures was $5 \mathrm{~cm}$. below the sternal angle, with the patient in the recumbent position. Immediately after pressure recording blood samples were drawn in the usual way and oxygen analyses were carried out using Van Slyke's method. To obtain pulmonary capillary arterial pressures the catheter was advanced through the right and left atrium and into a pulmonary vein under fluoroscopic control. The patient was then asked to take a deep breath, and the tip of the catheter was pushed further into the vein so as to obstruct its lumen.

\section{OBSERVATIONS}

In three of the five cases studied we made a diagnosis of pure atrial septal defect, in one atrial septal defect with complete transposition of the great vessels, and in one atrial and ventricular septal defect with dextrocardia, complete heart block, and probably complete transposition. The diagnosis was based on the results of catheterization, angiocardiography, and clinical findings. Since no case was suitable for surgical treatment the diagnosis could not be verified. 

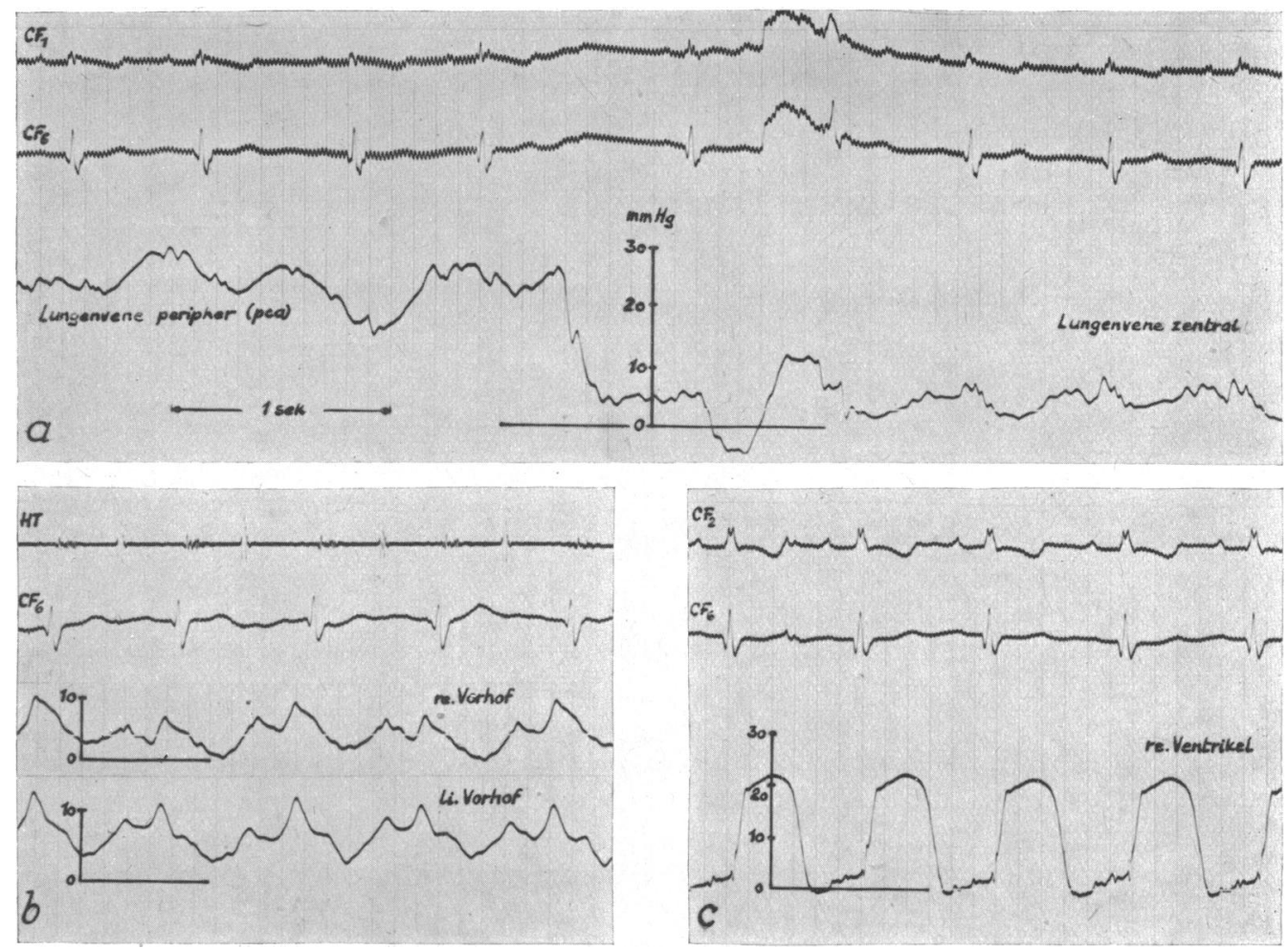

Fig. 1.-Case 1. (A) Withdrawal of the catheter from the occluded branch of a pulmonary vein under continuous pressure recording. (B) Superimposed tracings of the right and left atrial pressures. (C) Right ventricular pressure.
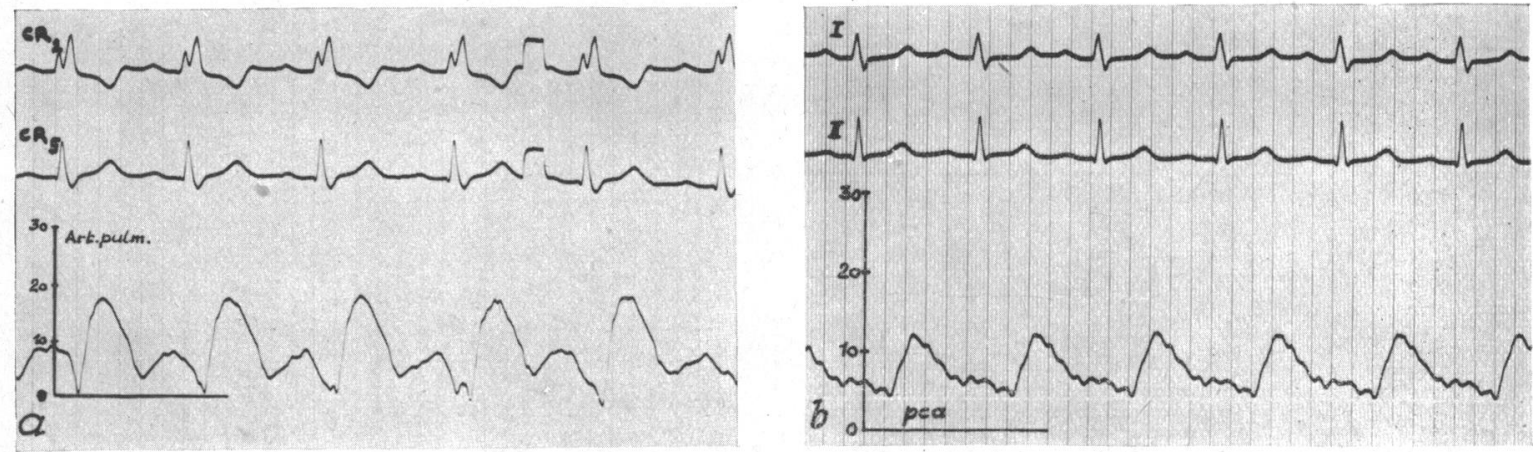

FIG. 2.-Case 3. (A) Pulmonary arterial pressure. (B) Pressure in the obstructed pulmonary vein.

Case 1. A girl, aged 9, with isolated atrial septal defect. The tracings of Fig. 1 were obtained in a right pulmonary vein, the catheter having been passed through the A.S.D. By obstructing a small branch of the vein, a curve resulted in which no venous elements can be recognized, its mean pressure being about $23 \mathrm{~mm}$. $\mathrm{Hg}$. Though disturbed by many artefacts these venous elements due to atrial contraction may be clearly seen after withdrawal of the catheter under continuous pressure recording. Simultaneously with the change in the shape of the curve, there occurs a sudden drop of pressure which is now in the mean about $5 \mathrm{~mm}$. $\mathrm{Hg}$. Pressures in the left and right atria were also recorded, and since the heart rate during both measurements was the same it was possible to superimpose both tracings. 
Case 2. A boy, aged 8, with atrial septal defect with complete transposition of the great vessels. Several pulmonary veins were entered. Again an "arterial" pressure pulse of $27 / 20 \mathrm{~mm}$. $\mathbf{H g}$ was obtained, and again a sudden drop of pressure simultaneously with the appearance of atrial waves was seen upon releasing the catheter tip from the obstructed venule. Attempts to enter the pulmonary artery arising in this case from the left ventricle failed.

Case 3. A woman, aged 20, with isolated atrial septal defect with gross dilatation of the pulmonary artery. An arterial tracing in the blocked pulmonary vein was obtained in this case with a lower systolic and a higher diastolic pressure than in the pulmonary artery (Fig. 2). The onset of the systolic pressure rise was delayed by about $0.1 \mathrm{sec}$. indicating that this pulsation was transmitted through the capillaries. A typical venous curve in an obstructed pulmonary artery has also been recorded, and its mean pressure was found to be well below the mean pressure in the obstructed pulmonary vein.

Case 4. A man, aged 18, with atrial and ventricular septal defect with dextrocardia, complete heart block, and probably complete transposition. By advancing the catheter through the saphenous vein, a left-sided inferior vena cava, and both atria, several pulmonary veins were entered. The tracings obtained in the right atrium, which received blood from all the pulmonary veins, showed high cannon waves due to simultaneous atrial and ventricular contractions. These and also the normal a-waves are absent in the PCA tracing superimposed in Fig. 3, its mean pressure now being well above the atrial mean pressure. High pressures were recorded in both ventricles, but the pulmonary artery could not be reached.

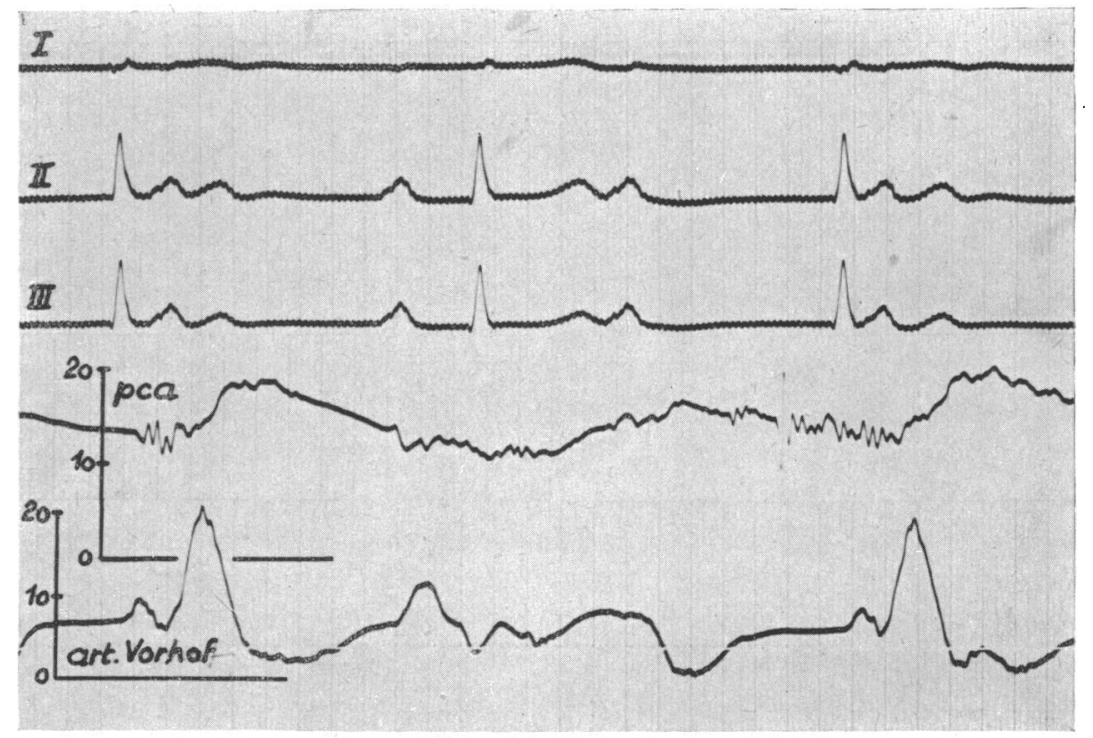

Fig. 3.-Case 4. Superimposed tracings from the blocked pulmonary vein and from the right atrium which was functionally the left.

Case 5. A boy, aged 16, with atrial septal defect and pulmonary hypertension and cyanosis. High pressures were found in the right ventricle and in the pulmonary artery presumably due to highly increased arteriolar resistance. Unfortunately, no attempts were made to enter the capillary bed from the arterial side. A " hæmodynamic alternans" will be recognized in the right ventricle, in the pulmonary artery, and also in the obstructed pulmonary vein (Fig. 4) thus proving the transmission of arterial pulsations through the capillaries. The time lag of the onset of the systolic pressure rise amounted to $0.07 \mathrm{sec}$. This was the only case where the mean pressure in the right atrium was higher than in the left. It may, therefore, be assumed that the apparent cyanosis was, at least partly, due to a predominant right to left shunt.

\section{Discussion}

As it will be seen from the tracings shown above the pressure pulsations obtained by occluding a small branch of the pulmonary vein (PCA) appear to have the following characteristics. (1) The shape of the curve resembles in many ways an arterial pulsation. (2) The mean pressure is higher in the obstructed than in the non-obstructed pulmonary vein and in both atria. (3) A considerable 

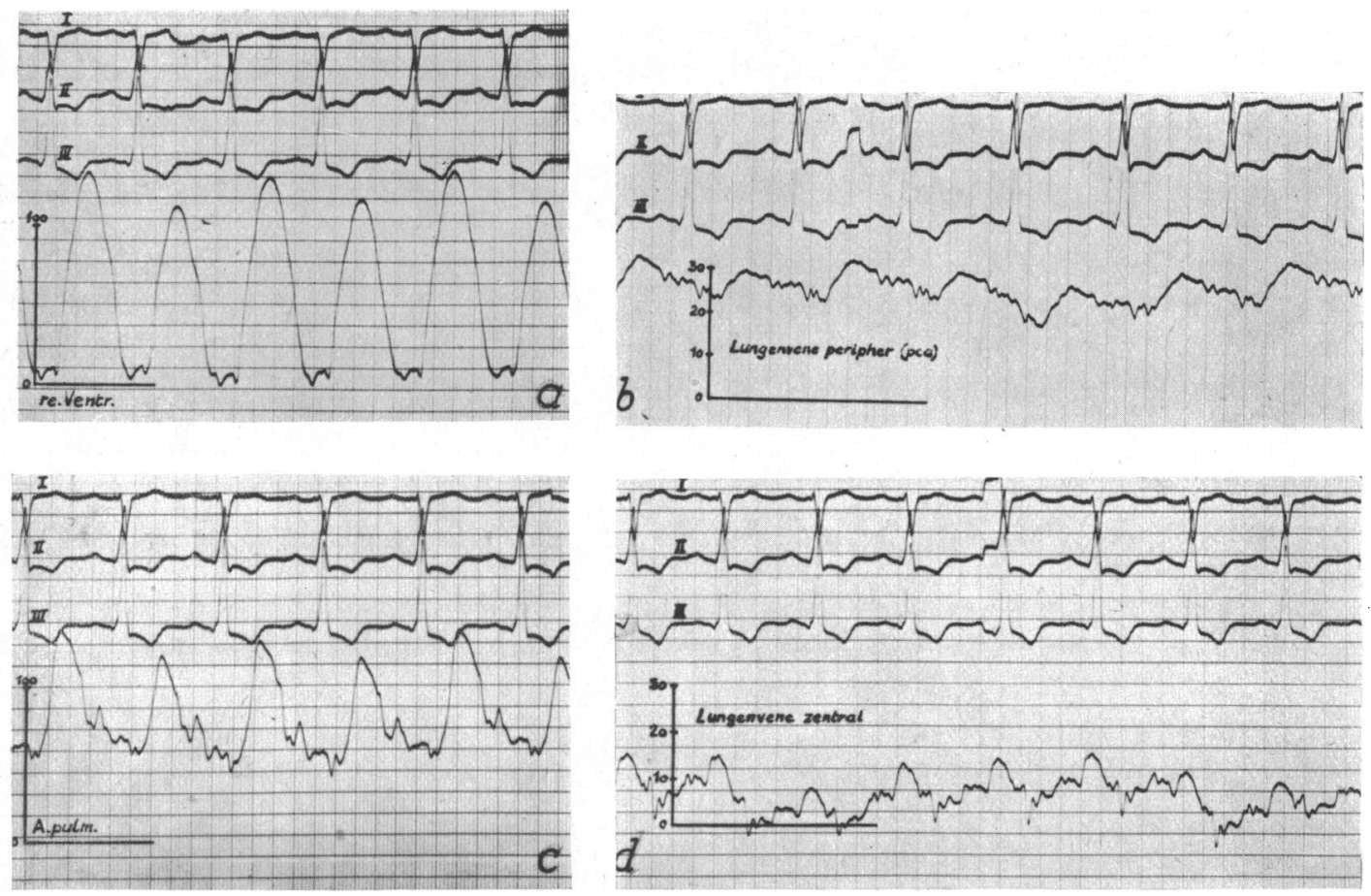

Fig. 4.-Case 5. (A) Right ventricular pressure. (B) Pressure in the obstructed pulmonary vein. Pulmonary arterial pressure.

(D) Pressure in the non-obstructed pulmonary vein.

(C)

time lag exists between the onset of the systolic pressure rise in the pulmonary artery and in the blocked pulmonary vein.

Further evidence for the PCA being a transmitted arterial pulsation has been brought by the observation that in one case showing high cannon waves in the non-obstructed pulmonary vein these could not be seen after obstructing it, and on the other hand, rhythmic variations of the systolic pressure height in the pulmonary artery due to a " hæmodynamic alternans" of the right ventricle have been recorded on the other side of the vascular bed.

In trying to interpret these results one has to recognize that the method of wedging a cardiac catheter into the capillaries is by no means a physiological one. An artificial manometric system is constructed which includes certain parts of the vascular bed and thus disturbs the normal flow and pressure conditions. Obstructing the blood flow on the venous side will result in a certain degree of congestion in front of the catheter tip. But since the capillary bed of the lung contains such a rich network of vessels that equilibrium should easily be adjusted by means of the neighbouring unobstructed capillaries maintaining undisturbed blood flow, this congestion seems unlikely to contribute much to the pressure height and cannot be the only reason for the arterial pulsations and the sudden pressure rise occurring after blocking only a very small branch of the pulmonary vein. To explain these findings we must assume that not only the capillaries but also the arterioles on the other side of the obstruction take part in the manometric system thus constructed. Then, the explanation offered for the venous pulsations observed after blocking a small branch of the pulmonary artery would be that in this case also the venules are included in the pressure conducting system.

It was recently reported by Calazel et al. (1951) that they were not able to obtain venous tracings in the obstructed pulmonary artery in five out of six patients, and thus could not confirm the findings of Lagerloef and Werkoe (1949) who stated that their PCV tracings closely resembled the pattern 
of the right atrial curves. Using the same manometric system and the same electrical damping as the Swedish workers, we should like to say that in most cases in which we tried to obtain PCV tracings we found rather distinct venous curves in the blocked pulmonary artery. Therefore, the failure of Calazel et al. in getting a "capillary" pulse resembling atrial tracings may well be due to the different recording and damping system they used.

We should like to think that the results reported by Lagerloef and Werkoe (1949) and in this paper, support the view of Cournand (1950) that the term "capillary" pressure commonly used for the mean pressure obtainable by blocking the pulmonary artery, is open to question; it is not only the pressure in the capillary bed but also the pressure in the venules on its other side one records by this method, and it is not only the "capillary" pressure but also the pressure in the arterioles one obtains by blocking the pulmonary vein. Otherwise the pressures thus recorded on both sides would be the same.

Identical mean pressures on both sides of the capillary bed were reported by Hellems, Haynes, and Dexter (1949) in two cases of atrial septal defect but could not be found by Calazel et al. who recorded in two similar cases PCA mean pressures that were almost twice as high as the PCV mean pressures. This difference was also found in one of our cases and was certainly of the same order in the other cases as well. Since it is now widely held that the "capillary" pressure gives an indication of the left atrial mean pressure, it should be emphasized that an error in the determination of the "capillary" pressure of only $1 \mathrm{~mm}$. $\mathrm{Hg}$ will usually result in an error in the range of 10 up to 25 per cent and this will enter into the various calculations which are now used for the determination of pulmonary pressure gradients, pulmonary arteriolar resistance, and mitral valve area (Dexter et al., 1950; Gorlin and Gorlin, 1951).

If we assume that the PCA tracings are arterial pulsations transmitted through the capillaries there is no doubt that the pulsations from the bronchial artery should considerably contribute to the pressure height in the blocked pulmonary vein. Therefore, we were not surprised to find no relationship between the PCA pressures and the pressures in the pulmonary artery or in the right ventricle, respectively. As it will be seen in the Table, in one case the PCA systolic and mean pressures were even higher than the systolic pressure in the right ventricle but since some time had elapsed between the two measurements during which the pressure in the right ventricle might have fallen, no decisive conclusions could be drawn.

TABLE

Data obtained in the Obstructed and Non-obstructed Pulmonary Veins, Pulmonary Arteries, AND RIGHT VENTRICLES

\begin{tabular}{|c|c|c|c|c|c|c|}
\hline \multirow{2}{*}{$\begin{array}{l}\text { Case } \\
\text { No. }\end{array}$} & \multicolumn{4}{|c|}{ Pressures in $\mathrm{mm} . \mathrm{Hg}$} & \multicolumn{2}{|c|}{$\begin{array}{c}\mathrm{O}_{2} \text { Saturations } \\
\text { (percentage) }\end{array}$} \\
\hline & $\begin{array}{l}\text { PV } \\
\text { m. }\end{array}$ & $\begin{array}{l}\text { PCA } \\
\text { s. d. }\end{array}$ & $\begin{array}{l}\text { PA } \\
\text { s. }\end{array}$ & $\begin{array}{l}\text { RV } \\
\text { s. } \quad \text { d. }\end{array}$ & PV & PCA \\
\hline $\begin{array}{l}1 \\
2 \\
3 \\
4 \\
5\end{array}$ & $\begin{array}{l}5 \\
8 \\
2 \\
8 \\
6\end{array}$ & $\begin{array}{rr}27 & 21 \\
27 & 20 \\
13 & 4 \\
17 & 12 \\
33 & 23 \\
(29) \dagger & \end{array}$ & $\begin{array}{c}{ }^{-} \\
18-1 \\
130-56 \\
(115) \dagger\end{array}$ & $\begin{array}{cc}22 & 0 \\
37^{*} & 7 \\
25 & 0 \\
125 \dagger & 4 \\
130 & 5 \\
(110) \ddagger & \end{array}$ & $\begin{array}{l}74 \cdot 2 \\
85 \cdot 6 \\
83 \cdot 0 \\
91 \cdot 0 \\
87 \cdot 3\end{array}$ & $\begin{array}{c}72 \cdot 8 \\
85 \cdot 6 \\
\overline{87 \cdot 3} \\
-\end{array}$ \\
\hline
\end{tabular}

Since a certain degree of dilatation of the pulmonary capillaries, which would facilitate the transmission of arterial pulsations, seems to be persistent with atrial septal defect, these results obtained in pathological cases cannot be readily applied to normal conditions. Considering further the nonphysiological method with which these results were obtained, no claim of significance upon the true hæmodynamic conditions on the arterial side of the pulmonary vascular bed should be made. 
There is one further point. As was shown by Dexter et al. (1946), fully oxygenated blood can be drawn from the capillary bed after blocking the pulmonary artery. It seemed, therefore, of interest to compare the degree of oxygen saturation in the samples drawn from the obstructed and non-obstructed pulmonary veins (see Table). In only three cases was it possible to obtain blood in the PCA position, and in two of them saturation was found to be slightly lower than in the nonobstructed vein. But before discussing the significance of these findings, we should like to wait till further confirmatory results have been gathered. A certain degree of unsaturation of the pulmonary venous blood was found in all patients but this seems to be quite common with atrial septal defect (Hickam, 1949; Wood, personal communication).

\section{SUMMARY}

In five cases of atrial septal defect, pressures on the venous side of the pulmonary capillary bed were recorded after wedging a cardiac catheter into a pulmonary vein so as to obstruct its lumen.

Since the tracing thus obtained closely resembles arterial pulsation it is called the " pulmonary capillary arterial pressure pulse."

It is assumed that these pulsations are transmitted through the capillaries from the arterial side of the pulmonary vascular bed.

In all cases the mean pressures in this position were found to exceed considerably the mean pressures in the non-obstructed pulmonary vein and in both atria.

\section{REFERENCES}

Calazel, P., Gerard, R., Daley, R., Draper, A., Foster, J., and Bing, R. J. (1951). Bull. Johns Hopk. Hosp., 88, 20. Cournand, A. (1950). Circulation, 2, 641.

Dexter, L., Burwell, C. S., Haynes, F. W., and Seibel, R. E. (1946). J. Clin. Invest., 25, 913.

Dow, J. W., Haynes, F. W. Whittenberger, J. L., Ferris, B. G., Goodale, W., and Hellems, H. K. (1950). J. Clin. Invest., 29, 602.

Gorlin, R., and Gorlin, S. G. (1951). Amer. Heart J., 41, 1.

Hellems, H. K., Haynes, F. W., Dexter, L., and Kinney, T. D. (1948). Amer. J. Physiol., 155, 98.

-, , (1949). J. Appl. Physiol., 2, 24.

Hickam, J. B. (1949). Amer. Heart J., 38, 801.

Lagerloef H., and Werkoe, L. (1949). Scand. J. Clin. Lab. Invest., 1, 147. 